\title{
Comparing the Efficacy of Dexmedetomidine When Used as an Adjuvant to Bupivacaine in Supraclavicular Brachial Plexus Blocks under Ultrasound-Guided
}

\author{
Elsayed Mohamed Abdelzaam (1) \\ Faculty of Medicine, Benha University, Banha, Egypt \\ Email: sayedzim12@yahoo.com
}

How to cite this paper: Abdelzaam, E.M. (2019) Comparing the Efficacy of Dexmedetomidine When Used as an Adjuvant to Bupivacaine in Supraclavicular Brachial Plexus Blocks under Ultrasound-Guided. Open Journal of Anesthesiology, 9, 99-109. https://doi.org/10.4236/ojanes.2019.95010

Received: April 8, 2019

Accepted: May 19, 2019

Published: May 22, 2019

\section{Copyright $\odot 2019$ by author(s) and} Scientific Research Publishing Inc. This work is licensed under the Creative Commons Attribution International License (CC BY 4.0). http://creativecommons.org/licenses/by/4.0/ Open Access

\begin{abstract}
Background: Ultrasound-guided for regional anesthesia offers many potential benefits in the emergency setting. Analgesia can be explicitly targeted to the region of pain and provide relief for many hours and decrease needing to the large volume of local anesthetic. The aim of the work: Comparing the efficacy of dexmedetomidine when used as an adjuvant to bupivacaine in supraclavicular brachial plexus blocks on the onset of sensory, motor blockade and postoperative analgesia. Patients and methods: This prospective, randomized, single-blind clinical study conducted on 60 patients underwent upper limb surgery done by ultrasound-guided supraclavicular brachial plexus block; these patients allocated into two equal groups: Group I (control) received $20 \mathrm{ccs}$ ( $19 \mathrm{cc}$ bupivacaine $0.5 \%+1 \mathrm{cc}$ saline), Group II received $20 \mathrm{cc}$ (19 cc bupivacaine $0.5 \%+1 \mathrm{cc}$ volume of Dexmedetomidine $1 \mathrm{ug} / \mathrm{kg}$ ). Results: Demographic data and surgical characteristics were comparable in both groups. The onset times for sensory and motor blocks were significantly shorter in Group II than Group I ( $\mathrm{P}<0.001)$, while the duration of blocks was considerably longer $(\mathrm{P}<0.001)$ in Group II. Except for the first recordings (at 0,5 , and $10 \mathrm{~min}$ ), heart rate levels in Group II were significantly lower $(\mathrm{P}<0.001)$. MBP levels in Group II at 15, 30, 45, 60, 90 and 120 min were significantly lower than in Group I $(\mathrm{P}<0.001)$. The duration of analgesia (DOA) was significantly longer in Group II than Group I ( $<<0.001)$. As regards to the visual Analouge score, there is a highly significant difference at 6 hours, 8 hours and 10 hours in Group II than Group I. Conclusion: We recommend adding Dexmedetomidine to local anesthetics in peripheral nerve blocks to take advantage of the prolonged time of both sensory and motor blocks and prolonged postoperative analgesia.
\end{abstract}




\section{Keywords}

Dexmedetomidine, Bupivacaine, Supraclavicular Brachial Plexus Blocks, Ultrasound-Guided

\section{Introduction}

Brachial plexus nerve block has many potential advantages, including optimal pain control, reduced use of opioids, and facilitation of early discharge after ambulatory procedures [1].

Successful brachial plexus blocks rely on proper techniques of nerve localization, needle placement, and local anesthetic injection.

Conventional approaches used today, unfortunately, are all (blind) techniques that rely on surface landmarks before needle insertion, and elicitation of paraesthesia or nerve stimulated muscle contraction after needle insertion. Often multiple trial-and-error needle attempts are necessary resulting in procedure-related pain and complications [2].

This is risky, particularly for the supraclavicular approach, because of the chance of pneumothorax. When brachial plexus blockade done under ultrasound guided, leading to provide images of the plexus, surrounding structures, and also allowing real-time guidance of the needle to the nerve target. Ultrasound guidance for brachial plexus blocks can permit the accurate deposition of the local anesthetic solution under constant observation and also improve success rates and decrease the incidence of complication [3].

Current ultrasound equipment allows much easier identification of tiny neural structures than it was possible with machines introduced only a few years ago. In addition, adjacent anatomical structures can be identified. The speed of ultrasound waves through biological tissue is based on the density of tissues, and not the frequency of the ultrasound waves. The higher the tissue density, the faster the ultrasound waves will travel [4].

Dexmedetomidine is a stereoisomer of medetomidine, with chemical formula [(1S)-1-(2,3-dimethylphenyl)ethyl]-1H-imidazole. It is a highly selective $\alpha 2$-adrenergic receptor (AR) agonist with a relatively high ratio of $\alpha 2 / \alpha 1$-activity (1620:1 as compared to 220:1 for clonidine) [5].

Regional anesthesia has been believed as one of the formats for effective perioperative pain control. Regional blocks using ultrasound-guided have become a perfect supplement to general anesthesia for extending analgesia. Brachial plexus nerve blockade has many benefits, including, reduced use of opioids, optimal pain control, and makes a better range of motion after joint surgery, and promotion of early discharge [6].

Ultrasound guidance offers several potential advantages, providing, direct visualization of nerve direct visualization of anatomical structures, allowing real-time control of needle advancement to the nerve target, avoidance of muscle 
twitches and permitting the accurate deposition of the local anesthetic solution under constant observation. At interscalene, supraclavicular, infraclavicular, and axillary levels, as well as distally in the arm, ultrasound has been used to guide peripheral nerve blockade [7].

Dexmedetomidine undergoes almost complete hydroxylation through direct glucuronidation and cytochrome P450 metabolism in the liver. Metabolites are excreted in the urine (about 95\%) and feces (4\%). The elimination half-life is approximately two hours. The average protein binding is $94 \%$. Effects of dexmedetomidine can be antagonized easily by administering the $\alpha 2$-AR antagonist atipamezole [8].

Dexmedetomidine is a strong sedative, analgesic, and it has anesthetic effects when used in general anesthesia, also, when it used as a perineural adjuvant to facilitate better anesthesia and analgesia [9].

The mechanism of action of dexmedetomidine by inhibits the function of sodium channels and neuronal potassium current which lead to blocking the hyperpolarization-activated cyclic nucleotide-gated channels, resulting in enhancement of activity-dependent hyperpolarization and also it the inhibited release of substance $p$ at dorsal root neuron [10].

We test the effect of adding dexmedetomidine to bupivacaine for supraclavicular brachial plexus block our primary endpoint will be the onset time and duration of motor and sensory blocks.

\section{Patients and Methods}

This randomized, single-blind, comparative study was performed after obtaining approval from the Ethics Committee of the hospital and written informed consent from sixty patients. Patients were allocated into two same groups. This study was carried out in the anesthesia department, faculty of medicine, Benha university hospitals from August 2017 to August 2018, randomization was done into two groups by lottery method. Those with history or presence of cardiac, inspiratory or renal failures, infection at the site of block, coagulopathy and those who are pregnant were not included in the study. Patients were randomized into two equal groups. The participants were randomly assigned into two groups 30 in each by a random sequence number produced by the computer and kept in sealed envelopes. The study investigator opened the closed envelopes. The all patients were with ASAI and II and their ages ranged between 18 to 60 . Before surgery, the participants received learning about the VAS pains core (0 10) and the technique and details of the nerve block techniques. Patients undergoing elective bony orthopedic surgeries in the upper limb under supraclavicular brachial plexus block. These sixty Patients were divided into two same groups; both groups receive ultrasound-guided supraclavicular brachial plexus block.

1) Group I ( $n=30)$ : patients were given a total $20 \mathrm{cc}$ solution consisting of 19 cc bupivacaine $0.5 \%$ with one cc of isotonic sodium chloride solution.

2) Group II $(n=30)$ : All the patients in this group given $20 \mathrm{cc}$ solution con- 
sisting of $19 \mathrm{cc}$ bupivacaine $0.5 \%$ with a $1 \mathrm{cc}$ volume of $1 \mathrm{ug} / \mathrm{kg}$ dexmedetomidine (Dexmedetomidine hydrochloride (Precedex ${ }^{\oplus}$, supplied in $100 \mu \mathrm{g} /$ manufactured by Hospira, Inc. Lake Forest, IL, USA) plus normal saline.

Before starting the block, when the patient's arrival to the operating room, a $20 \mathrm{G}$ intravenous scannula inserted in the healthy arm, preoperative fluid requirements were calculated and administered throughout the procedure. All patients were monitored with ECG, non-invasive blood pressure monitoring and pulse oximetry and baseline measurement were recorded Patients were given 1 $2 \mathrm{mg}$ of midazolam intravenous (IV) as a premedication 10 - $15 \mathrm{~min}$ before beginning block technique in addition to $50-100 \mu \mathrm{g}$ of fentanyl just prior to block needle insertion; patient lying supine and the head turned $45^{\circ}$ to the contralateral side.

The technique of ultrasound guided supraclavicular brachial plexus block:

After injecting a small amount of $1 \%$ lidocaine to anesthetize the skin, insert the short bevel needle along the longitudinal axis of the ultrasound probe (in-plane needle approach). Ensure the needle is kept parallel to this axis at all times to improve visualization of the needle tip. First to locate the subclavian artery in the short axis view, where the artery appears as a round pulsating, hypoechoic structure. The key to safety and success with the supraclavicular approach is visualizing the anatomy of the brachial plexus in three dimensions in which the trunks of the brachial plexus are horizontally arranged at the border of the sternocleidomastoid, lie posterior to the subclavian artery, are superior to the lung medially, and superior to the first rib laterally. An essential objective for ultrasound is a visualization of the spread of local anesthetic during the injection. Confirmation of the correct disposition of local anesthetic avoids any maldistribution, such as epineural, perineural, or intravascular injection. Besides, an ability to perform blocks with small volumes of local anesthetic is mainly based on an ability to observe the spread of the local anesthetic.

\section{Measurements}

- Onset time for the sensory block: was defined as the time interval between the end of local anesthetic administration and complete sensory neighborhood (score 2 for all nerves).

- Duration of the sensory block: is detected as the time interval between the complete sensory block to complete recovery which detected by cold and pain sensation that tested by an alcohol swab and pinprick in all nerves.

- Onset time for the motor block: was defined as the time interval between total local anesthetic administration and complete motor block (Grade 2).

- Duration of the motor block: is also detected by the time interval from full motor block to the motor function of hand and forearm become complete recovery (Grade 0 ).

- Duration of analgesia (DOA): This defined by the time which starts from the complete sensory block to the first analgesic request was recorded. 
- Hemodynamic parameters: mean arterial blood pressure (MAP) and heart rate were noted then recorded.

- VAS scale: $(0=$ no pain and $10=$ worst imaginable pain $)$ to estimate the pain at rest in the recovery room and at 2, 4, 6, 8, 10, 12 hours after surgery. All complications recorded like hypotension (i.e. $20 \%$ decrease relative to baseline), bradycardia ( $\mathrm{HR}<50$ beats $/ \mathrm{min}$ ), nausea, vomiting, and hypoxemia $(\mathrm{spO} 2<90 \%)$.

\section{Data Management and Statistical Analysis}

The data were analyzed using Statistical Package for the Social Sciences version 21.0 (SPSS Inc. Chicago, Illinois, USA). The categorical variables are given in numbers and percentage (\%), and the continuous variables are given as mean \pm SD. The Kolmogorov-Smirnov test tested the normality of data. The quantitative variables were compared using the Unpaired t-test or Mann-Whitney test (when the datasets were not normally distributed) between the groups. The qualitative variables were compared using the Chi-square test or Fisher's exact test. $\mathrm{P}<0.05$ was considered statistically significant.

\section{Results}

Sixty patients completed the study. Table 1 was summarized all demographic data. No significant difference between both groups as regard, sex, age, height, ASA physical status weight, and surgical duration.

When comparing heart rate between both groups (Figure 1), the current study showed a significantly lower heart rate (HR) values in Group II at $15 \mathrm{mi}-$ nutes, $30 \mathrm{~min}$, $45 \mathrm{~min}, 60 \mathrm{~min}, 90 \mathrm{~min}$ and $120 \mathrm{~min}$ from bolus dose injection, compared to Group I.

Table 1. Demographic characteristics of patients data were presented as mean \pm SD Sex and ASA data were presented as numbers and percentage.

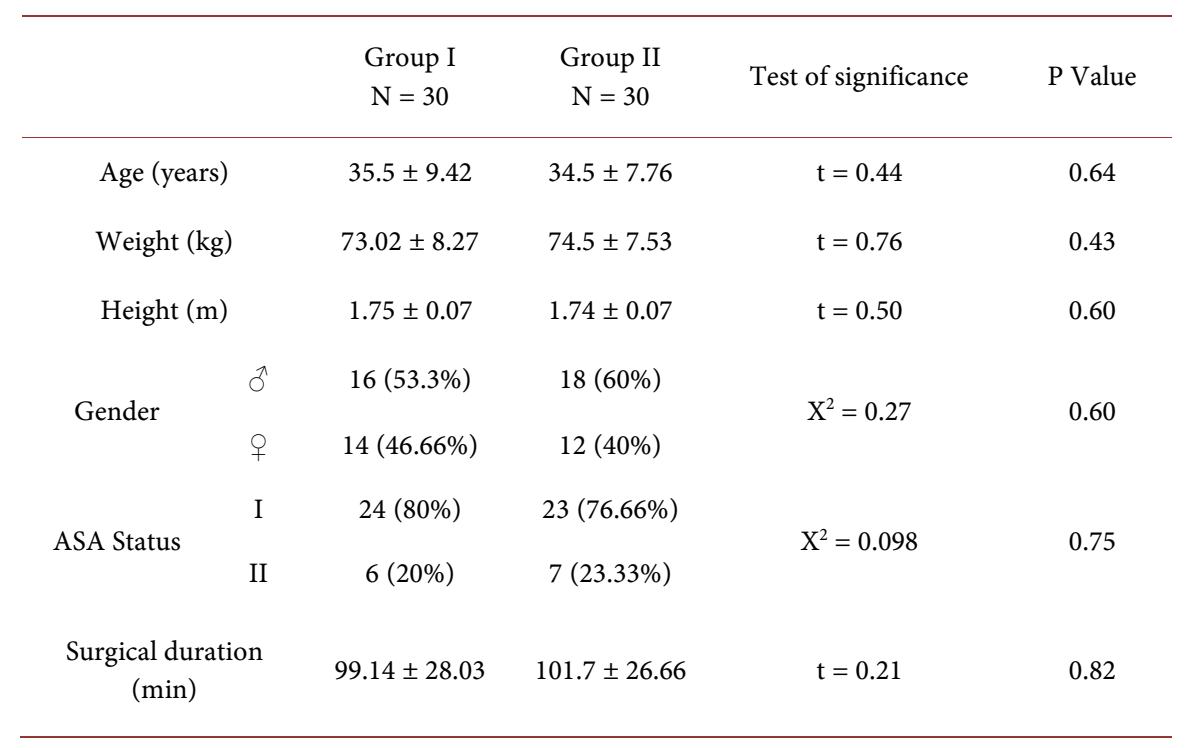


HR

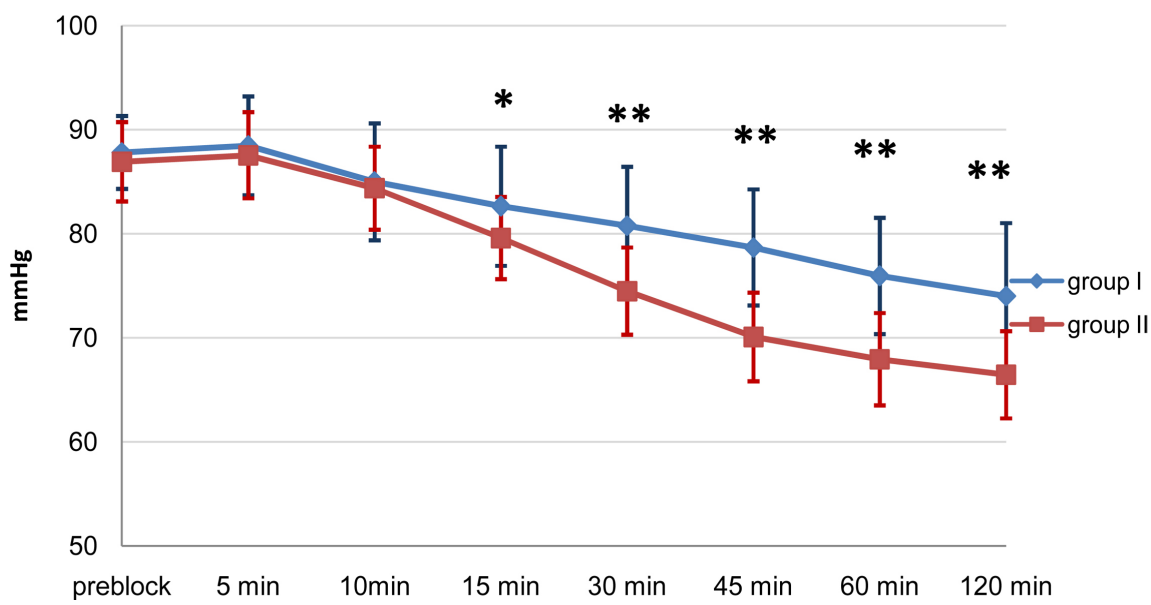

Figure 1. Comparison between both groups as regards heart rate. ${ }^{\star}$ significant. ${ }^{*}$ Highly significant.

As views comparing mean arterial blood pressure (MAP) between both groups, the current study showed a significantly lower MAP value in Group II at $15 \mathrm{~min}, 30 \mathrm{~min}, 45 \mathrm{~min}, 60 \mathrm{~min}$, and $120 \mathrm{~min}$ from bolus dose injection (Figure 2).

As regarding sensory block onset and duration; there is a highly significant difference between both groups and also motor block onset and length and duration of analgesia, this show in Table 2 . The onset times for sensory and motor blocks were significantly shorter in Group II than Group I $(\mathrm{P}<0.001)$, while the duration of blocks was significantly longer $(\mathrm{P}<0.001)$ in Group II.

As regards the visual analouge score, there is a highly significant difference between both groups at 6 hours, 8 hours and 10 hours (Figure 3). At two hours postoperatively, there was no statistical difference between both groups as regard VAS where $(P>0.05)$. At four hours postoperatively, there was no statistical difference between groups as regard VAS where $(\mathrm{P}>0.05)$. At six hours postoperatively, VAS was $1.60 \pm 0.56$ in group I and was $1.03 \pm 0.18$ in group II, and there was a statistically high significant increase in group I where $(\mathrm{P}<0.001)$. At eight hours postoperatively, VAS was $3.43 \pm 0.66$ in group I and was $1.3 \pm 0.60$ in group II, and there was a statistically high significant increase in group I where $(\mathrm{P}<0.001)$. At ten hours postoperatively, VAS was $3.63 \pm 0.49 \mathrm{i}$ group I and was $2.26 \pm 0.44$ in group II, and there was a statistically high significant increase in group I where $(\mathrm{P}<0.001)$. At twelve hours postoperatively, VAS was $4.53 \pm 0.50$ in group I and was $4.43 \pm 0.50$ in group II, and there was a statistically non significant increase where $(\mathrm{P}>0.05)$.

\section{Discussion}

The primary target of postoperative care is to minimize postoperative pain, nausea, vomiting and enhance early ambulation to prevent the development of venous thromboembolism and pneumonia. There is an advance in acute postoperative 


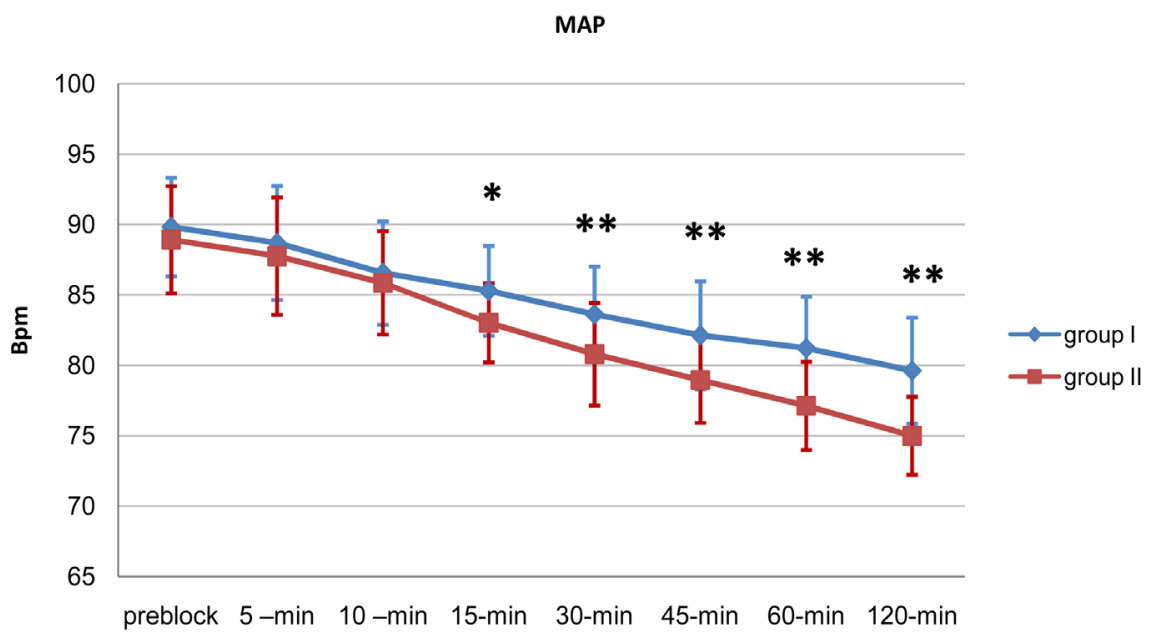

Figure 2. Comparison between both groups as regards mean Blood Pressure (MAP). ${ }^{\star}$ significant. ${ }^{*}$ Highly significant.

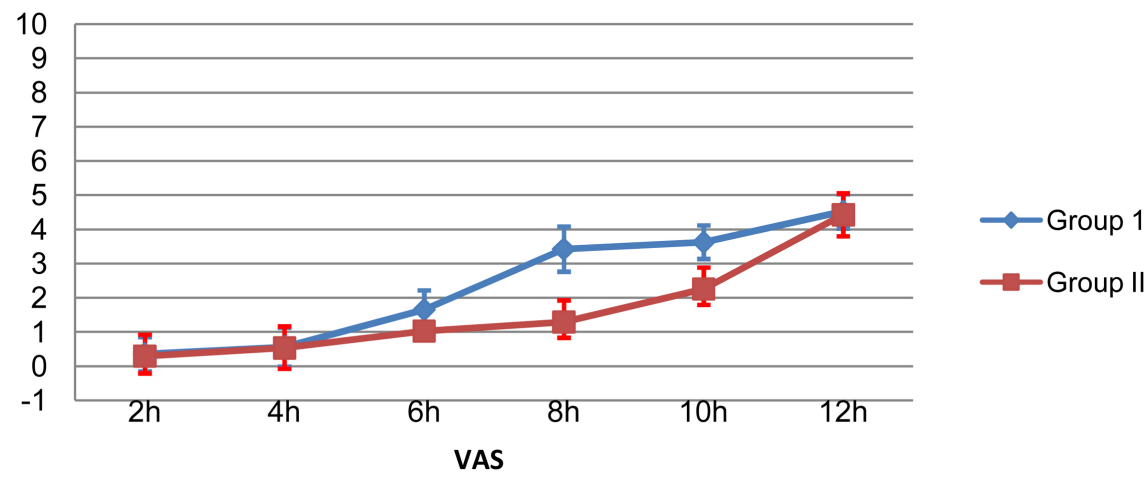

Figure 3. Comparison between visual analogue score (VAS) of both groups.

Table 2. Comparing both groups as regard sensory block, motor block.

\begin{tabular}{cccccc}
\hline \multirow{2}{*}{ Sensory block } & $\begin{array}{c}\text { Group I } \\
\mathrm{N}=30\end{array}$ & $\begin{array}{c}\text { Group II } \\
\mathrm{N}=30\end{array}$ & t-test & p-value \\
& Onset & $17.57 \pm 6.61$ & $12.91 \pm 2.01$ & 6.53 & $<0.001$ \\
& Duration & $322.36 \pm 51.76$ & $571.06 \pm 44.54$ & 19.92 & $<0.001$ \\
\multirow{2}{*}{ Motor block } & Onset & $23.62 \pm 3.05$ & $16.87 \pm 2.6$ & 9.33 & $<0.001$ \\
& Duration & $281.83 \pm 56.51$ & $535.73 \pm$ & 20.7 & $<0.001$ \\
\multirow{2}{*}{ Duration of analgesia } & $371.50 \pm 47.13$ & $692.32 \pm 83.58$ & 18.37 & $<0.001$ \\
\hline
\end{tabular}

pain management from patient controlled opioid analgesia, but at risk of its side effects and from parental NSAIDS with their abiliting to reduce postoperative dynamic pain [11].

Ultrasound-guided for regional anesthesia offers many potential benefits in the emergency setting. Analgesia can be explicitly targeted to the region of pain and provide relief for many hours and decrease needing to the large volume of local anesthetic. Besides, by providing an alternative to procedural sedation, there seems to the reduced hospital stay, shortened post-procedure observation 
periods with reduced need for nursing care, and high patient satisfaction [12].

In our study, show the following data, Demographic data and surgical characteristics were comparable in both groups. As regard age, sex, height, weight, surgical duration, and ASA physical status, there was no significant difference between groups. As regards comparing heart rate (HR) between both groups, the current study showed a significantly lower heart rate (HR) values in group II at 15.

Minutes, $30 \mathrm{~min}, 45 \mathrm{~min}, 60 \mathrm{~min}, 90 \mathrm{~min}$ and $120 \mathrm{~min}$ from bolus dose injection, compared to Group I. As views comparing mean arterial blood pressure (MAP) between both groups, the current study showed a significantly lower MAP value in Group II at $15 \mathrm{~min}, 30 \mathrm{~min}, 45 \mathrm{~min}, 60 \mathrm{~min}$, and $120 \mathrm{~min}$. The onset times for sensory and motor blocks were significantly shorter in Group II than Group I ( $\mathrm{P}<0.001)$, while the duration of blocks was significantly longer $(\mathrm{P}<0.001)$ in group II. As regards the visual analouge score, there is a highly significant difference between both groups at 6 hours, 8 hours and 10 hours At six hours postoperatively, VAS was $1.60 \pm 0.56$ in group I, and was $1.03 \pm 0.18$ in Group II, there was a statistically high significant increase in Group I where $(\mathrm{P}<$ $0.001)$. At eight hours postoperatively, VAS was $3.43 \pm 0.66$ in Group I and was $1.3 \pm 0.60$ in Group II, and there was a statistically high significant increase in Group I where $(\mathrm{P}<0.001)$. At ten hours postoperatively, VAS was $3.63 \pm 0.49 \mathrm{i}$ Group I and was $2.26 \pm 0.44$ in group II, and there was a statistically highly significant increase in Group I where $(\mathrm{P}<0.001)$.

El-Hennawy AM, et al., shown, the $\alpha 2$-adrenoceptors (ARs) by virtue of their sedative, analgesic, sympatholytic, anesthetic-sparing and hemodynamic-stabilizing properties, have been used as an adjunct to local anesthetics for prolongation of effect. Clonidine, an 02 -AR agonists has been used with local anesthetics in peripheral nerve block, though the results have been somewhat less impressive [13]. Rancourt MP, et al., demonstrate dexmedetomidine, a highly selective $\alpha 2$-AR agonist has recently been introduced in anesthesia practice. It is currently being used for continuous intravenous sedation in the intensive care setting, and procedural sedation in non intubated patients. Its potential benefit as an adjuvant to local anesthetics in peripheral nerve blocks has emphasized in a few experimental studies [14].

Some recent studies show the effects of mixing dexmedetomidine with local anesthetics during peripheral nerve and nerve plexus blockade. A survey by Obayah and colleagues added Dexmedetomidine to bupivacaine during placement of a more great palatine nerve block for cleft palate repair [15].

When dexmedetomidine added to bupivacaine provided lower pain scores and prolonged analgesia (approximately 50\%) with no adverse effect on hemodynamics when compared with bupivacaine alone, another study by Esmaoglu et al. show that dexmedetomidine with levobupivacaine during placement of axillary brachial plexus blockade that provided in decreasing of onset block time and longer block duration resulting in improved postoperative analgesia [16]. 
Also, there is several animal studies have investigated the analgesic effects of dexmedetomidine as an adjunct. This study performed on a rat model by Brummett and colleagues reported that dexmedetomidine added to ropivacaine during sciatic nerve blockade provided more extended analgesia than systemic administration [17].

Another sciatic nerve rat model investigation when dexmedetomidine is adding to bupivacaine assessed its efficacy and safety. In this study, they discovered that prolonged duration of sensory and motor blockade, when adding dexmedetomidine to bupivacaine but, dexmedetomidine alone do not show any evidence of significant in duration sensory and motor blockade, resulted in prolonged duration to thermal protection/analgesia in a dose-dependent manner [18].

Memis et al. in their study showed that when dexmedetomidine adding to lignocaine for intravenous regional anaesthesia, this leading to improves both the quality of anaesthesia as well as intraoperative and post-operative analgesia. In this study, the quality of block in $87 \%$ of the patients in Group D was grade IV, and $47 \%$ in Group K achieved grade IV quality [19].

kwon et al., evaluated the sedative effect of perineural dexmedetoedine for supraclavicular brachial plexus block by used the bispectral index and observed that it corresponds to a bispectral index value of 60 , from which patients are easily awakened in a lucid state [20].

Vandana $\mathrm{M}$ et al., show, Addition of dexmedetomidine to $0.75 \%$ ropivacaine in supraclavicular brachial plexus block significantly prolongs the duration of analgesia [21].

\section{Conclusion}

We recommend adding Dexmedetomidine to local anesthetics in peripheral nerve blocks to take advantage of the prolonged time of both sensory and motor blocks and prolonged postoperative analgesia.

\section{Conflicts of Interest}

The author declares no conflicts of interest regarding the publication of this paper.

\section{References}

[1] Kathuria, S. and Dhawan, I. (2015) Dexmedetomidine as an Adjuvant to Ropivacaine in Supraclavicular Brachial Plexus Block. Saudi Journal of Anaesthesia, 9, 148-154. https://doi.org/10.4103/1658-354X.152841

[2] Imani, F., Heamati, K., Rahimzadeh, P., Kazemi, M.R. and Hejazian, K. (2016) Effectiveness of Stellate Ganglion Block under Ultrasound Guidance in the Upper Extremity. Journal of Clinical and Diagnostic Research, 10, UC9-UC12. https://doi.org/10.7860/JCDR/2016/14476.7035

[3] Rahimzadeh, P. and Faiz, S.H. (2013) Ultrasound a New Paradigm in Regional Anesthesia Pain Management. Anesthesiology and Pain Medicine, 3, 228-229. https://doi.org/10.5812/aapm.13363 
[4] Ammar, A.S. and Mahmoud, K.M. (2012) Ultrasound-Guided Single Injection Infraclavicular Brachial Plexus Block Using Bupivacaine Alone or Combined with Dexmedetomidine for Pain Control in Upper Limb Surgery: A Prospective Randomized Controlled Trial. Saudi Journal of Anaesthesia, 6, 109-114. https://doi.org/10.4103/1658-354X.97021

[5] Mirkheshti, A., Saadatniaki, A., Salimi, A., Manafi Rasi, A., Memary, E. and Yahyaei, H. (2014) Effects of Dexmedetomidine versus Ketorolac as Local Anesthetic Adjuvants on the Onset and Duration of Infraclavicular Brachial Plexus Block. Anesthesiology and Pain Medicine, 4, e17620. https://doi.org/10.5812/aapm.17620

[6] Pavan, K., Raju, B.C. and Coventry, D.M. (2014) Ultrasound-Guided Brachial Plexus Blocks. Continuing Education in Anaesthesia Critical Care \& Pain, 14, 185-191. https://doi.org/10.1093/bjaceaccp/mkt059

[7] Elahi, F., Reddy, C. and Ho, D. (2015) Ultrasound-Guided Peripheral Nerve Stimulation Implant for Management of Intractable Pain after Inguinal Herniorrhaphy. Pain Physician, 18, E31-E38.

[8] Gertler, R., Brown, H.C., Mitchell, D.H. and Silvius, E.N. (2001) Dexmedetomidine: A Novel Sedative-Analgesic Agent. BUMC Proceedings, 14, 13-21.

https://doi.org/10.1080/08998280.2001.11927725

[9] Das, B., Lakshmegowda, M., Sharma, M., et al. (2016) Supraclavicular Brachial Plexus Block Using Ropivacaine Alone or Combined with Dexmedetomidine for Upper Limb Surgery: A Prospective, Randomized, Double-Blinded, Comparative Study. Revista Española de Anestesiología y Reanimación, 63, 135-140. https://doi.org/10.1016/j.redar.2015.04.012

[10] Gu, X.Y., Liu, B.L., Zang, K.K., et al. (2015) Dexmedetomidine Inhibits Tetrodotoxin-Resistant Nav1.8 Sodium Channel Activity through Gi/Co-Dependent Pathway in Rat Dorsal Root Ganglion Neurons. Molecular Brain, 8, 15. https://doi.org/10.1186/s13041-015-0105-2

[11] Kehlet, H. and Dahl, J.B. (1993) The Value of Multimodal or Balanced Analgesia in Postoperative Pain Treatment. Anesthesia \& Analgesia, 77, 1048-1056. https://doi.org/10.1213/00000539-199311000-00030

[12] Beaudoin, F.L., Nagdev, A., Merchant, R.C. and Becker, B.M. (2010) Ultrasound-Guided Femoral Nerve Blocks in Elderly Patients with Hip Fractures. The American Journal of Emergency Medicine, 28, 76-81. https://doi.org/10.1016/j.ajem.2008.09.015

[13] El-Hennawy, A.M., Abd-Elwahab, A.M., Abd-Elmaksoud, A.M., El-Ozairy, H.S. and Boulis, S.R. (2009) Addition of Clonidine or Dexmedetomidine to Bupivacaine Prolongs Caudal Analgesia in Children. Bitish Journal of Anaesthesia, 103, 268-274. https://doi.org/10.1093/bja/aep159

[14] Rancourt, M.P., Albert, N.T., Cote, M., Letourneau, D.R. and Bernard, P.M. (2012) Posterior Tibial Nerve Sensory Blockade Duration Prolonged by Adding Dexmedetomidine to Ropivacaine. Anesthesia \& Analgesia, 115, 958-962. https://doi.org/10.1213/ANE.0b013e318265bab7

[15] O bayah, G.M., Refaie, A., Aboushanab, O., Ibraheem, N. and Abdelaziz, M. (2010) Addition of Dexmedetomidine to Bupivacaine for More Great Palatine Nerve Block Prolongs Postoperative Analgesia after Cleft Palate Repair. European Journal of Anaesthesiology, 27, 280-284. https://doi.org/10.1097/EJA.0b013e3283347c15

[16] Esmaoglu, A., Yegenoglu, F., Akin, A. and Turk, C.Y. (2010) Dexmedetomidine, When Added to Levobupivacaine Prolongs Axillary Brachial Plexus Block. Anes thesia \& Analgesia, 111, 1548-1551. https://doi.org/10.1213/ANE.0b013e3181fa3095 
[17] Brummett, C.M., Hong, E.K., Janda, A.M., Amodeo, F.S. and Lydic, R. (2011) Perineural Dexmedetomidine, When Added to Ropivacaine for Sciatic Nerve Block in Rats to Prolongs the Duration of Analgesia by Blocking the Hyperpolarization-Activated Cation Current. Anesthesiology, 115, 836-843. https://doi.org/10.1097/ALN.0b013e318221fcc9

[18] Brummett, C.M., Padda, A.K., Amodeo, F.S., Welch, K.B. and Lydic, R. (2009) Perineural Dexmedetomidine, When Added to Ropivacaine, Causes a Dose-Dependent Increase in the Duration of Thermal Antinociception in Sciatic Nerve Block in the Rat. Anesthesiology, 111, 1111-1119. https://doi.org/10.1097/ALN.0b013e3181bbcc26

[19] Memphis, D., Turan, A., Karamanlioglu, B., Pamukçu, Z. and Kurt, I. (2004) Adding Dexmedetomidine to Lignocaine for IVRA. Anesthesia \& Analgesia, 98, 835-840.

[20] Kwon, Y., Hwang, S.M., Lee, J.J. and Kim, J.H. (2015) The Effect of Dexmedetomidine as an Adjuvant to Ropivacaine on the Bispectral Index for Supraclavicular Brachial Plexus Block. Korean Journal of Anesthesiology, 68, 32. https://doi.org/10.4097/kjae.2015.68.1.32

[21] Mangal, V., Mistry, T., Sharma, G., Kazim, M., Ahuja, N. and Kulshrestha, A. (2018) Effects of Dexmedetomidine When Used as an Adjuvant to Ropivacaine in Ultrasound-Guided Supraclavicular Brachial Plexus Block: A Prospective, Randomized, Double-Blind Study. Journal of Anaesthesiology Clinical Pharmacology, 34, 357-361. 